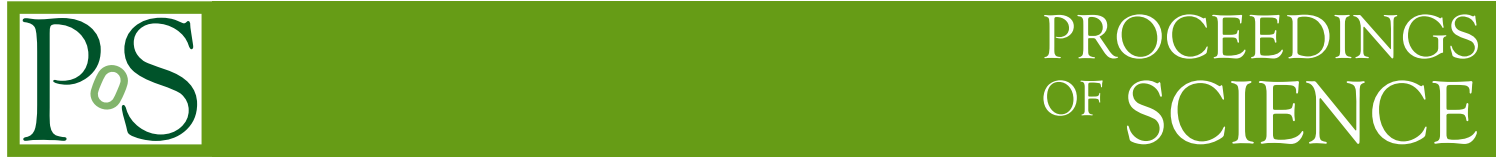

\title{
Standard Model Higgs Searches at the Tevatron
}

Kenneth Herner*

University of Michigan

On behalf of the CDF and DO Collaborations

E-mail: khernerefnal.gov

We report results of searches for the Standard Model Higgs Boson at the Fermilab Tevatron using up to $5.4 \mathrm{fb}^{-1}$ of data taken with the CDF and D0 detectors. There is no significant excess in the mass range of interest and the experiments set upper limits on the Higgs boson production cross section, including an exclusion of the Standard Model Higgs in the mass range 162-166 GeV.

XVIII International Workshop on Deep-Inelastic Scattering and Related Subjects, DIS 2010

April 19-23, 2010

Firenze, Italy

${ }^{*}$ Speaker. 


\section{Introduction}

The Higgs Boson, postulated to give mass to to the electroweak vector bosons, is the last unobserved particle in the Standard Model (SM). The Higgs mass is not theoretically predicted although direct searches and indirect searches via precision electroweak measurements yield a mass constraint of $114.4<m_{H}<186 \mathrm{GeV}$ at $95 \%$ Confidence Level (C.L.) [1]. It is precisely this mass range that can be probed at the Fermilab Tevatron. A resolution to the question of whether the Higgs exists is one of the central goals of the Tevatron physics program.

\section{Low-mass Higgs Searches}

Below a mass of $\approx 135 \mathrm{GeV}$ (the "Low-mass" region) the dominant Higgs decay is to a pair of $b$-quarks. Though direct observation of the gluon fusion process $g g \rightarrow H \rightarrow b \bar{b}$ is not feasible due to the overwhelming background, associated production with a $W$ or $Z$ is accessible at the Tevatron. The CDF and D0 collaborations both conduct searches for associated Higgs production. We summarize the most important low-mass searches below.

\section{1 $W H \rightarrow \ell v b \bar{b}$ Search}

$W H$ production has the largest cross section among the low-mass channels. Leptonic decays of the $W$ to $e v$ or $\mu v$ are the most sensitive sub-channels. CDF and D $\emptyset$ conduct searches in this channel with $4.8 \mathrm{fb}^{-1}$ and $5.0 \mathrm{fb}^{-1}$ of data, respectively [2, 3]. The final state includes a charged lepton $\left(e\right.$ or $\mu$ ), large missing transverse momentum $\left(B_{T}\right)$, and at least two jets. The leading backgrounds include $W / Z+$ jets production (including $W+$ heavy flavor jets), $t \bar{t}$ and single top production, and multijet production. Further selections are made to ensure that the $E_{T}$ and lepton are consistent with the decay of a $W$ boson. At this stage of the analysis the event selection is dominated by $W+$ jets events, as shown in Figure 1a for the $\mathrm{D} \emptyset$ analysis.

\section{2 $Z H \rightarrow \ell \ell b \bar{b}$ Search}

The $Z H \rightarrow \ell \ell b \bar{b}$ channel has a lower cross section, but has the advantage of being a fully reconstructed final state. Both $\mathrm{CDF}$ and $\mathrm{D} \varnothing$ search for events with two charged leptons consistent with the decay of a $Z$ boson and at least two jets $[4,5]$. The leading backgrounds are $Z+j e t s, t \bar{t}$ production, and multijet production. High lepton acceptance is important in this analysis and CDF and $D \emptyset$ extend the search to lepton+track final states to recover events where only the track from a second lepton is identified. Additionally the di-lepton and di-jet systems can be kinematically constrained and the di-jet invariant mass corrected, yielding a $10 \%$ sensitivity gain in the CDF analysis as shown in Figure $1 b$.

\section{3 $Z H \rightarrow v v b \bar{b}$ Search}

The $Z H \rightarrow v v b \bar{b}$ channel has a higher rate than $\ell \ell b \bar{b}$ due to the larger $Z$ branching fraction, but suffers from increased multijet backgrounds. This channel also receives a sizable contribution from $W H$ production when the lepton from the $W$ decay is not identified. CDF and D $\varnothing$ search for two jets and large $Z_{T}$ in $3.6 \mathrm{fb}^{-1}$ and $5.2 \mathrm{fb}^{-1}$, respectively $[6,7]$. Good rejection of the multijet background is required to have sensitivity in this channel. The two experiments exploit variables 
that have good separation between signal and background, such as the $\not_{T}$ significance (a measure of the likelihood that the measured $\not_{T}$ is from physical sources) shown in Figure 1c.

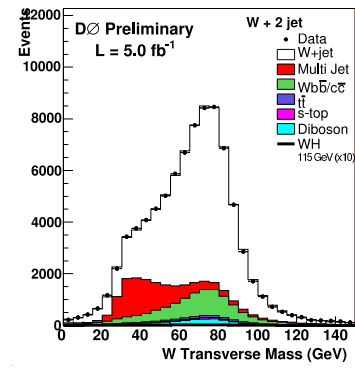

(a)

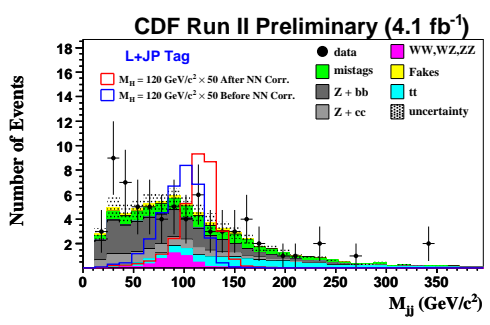

(b)

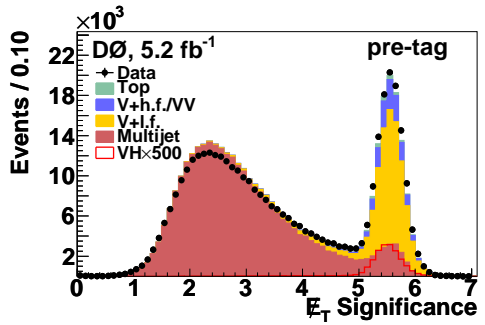

(c)

Figure 1: (a) $W$ transverse mass in the $\mathrm{D} \emptyset W H$ analysis. (b) Di-jet invariant mass in the CDF $Z H \rightarrow \ell \ell b \bar{b}$ analysis. (c) $Z_{T}$ Significance in the $\mathrm{D} \varnothing \mathrm{ZH} \rightarrow v v b \bar{b}$ analysis.

\section{$2.4 b$-tagging}

Since the Higgs decay is to a pair of $b$ quarks in this mass range robust $b$-jet identification is essential in this an all low-mass analyses for discriminating against the otherwise overwhelming backgrounds. The analyses described above have S/B ratios worse than 1:1000 before applying $b$-jet identification techniques. Both CDF and D $\varnothing$ employ "taggers" designed to identify jets likely to come from $b$ fragmentation using track, jet, and secondary vertex information. Such taggers are typically $50-60 \%$ efficient with misidentification rates of $\approx 1 \%$.

\subsection{Multivariate Techniques}

After $b$-tagging the S/B ratios in the low mass analyses are still on the order of $1 / 100$. Further discrimination is needed to reach SM sensitivity. Both experiments employ multivariate techniques to further separate potential signal and background. The CDF analysis uses $4.8 \mathrm{fb}^{-1}$ of data with an Event Probability Discriminant as the final variable discriminating against signal and background [2], while the $5.0 \mathrm{fb}^{-1} \mathrm{D} \emptyset$ analysis uses a neural network as its final discriminant. The $Z H \rightarrow \ell \ell b \bar{b}$ $\mathrm{CDF}$ analysis uses a neural network, while the $\mathrm{D} \emptyset$ analysis uses a boosted decision tree. The $Z H \rightarrow v v b \bar{b}$ analyses both use decision tree-based discriminants as the final variable.

\section{High-mass Higgs Searches}

Above a mass of $\approx 135 \mathrm{GeV}$ (the "High-mass" region) the dominant Higgs decay is to a pair of $W$ bosons, offering a clean final state when both $W$ bosons decay to $\ell v$. The signature is two oppositely-charged leptons with a large missing ET. Both CDF and DØ carry out searches in this channel, with the most important sub=channels being $\mu \mu \nu v, e e v v$, and $e \mu v v$. The most important backgrounds to high-mass searches are diboson production and Z/Drell-Yan production. A number of variables can be exploited to distinguish between signal and background, including the di-lepton invariant mass (Fig. 2a), and the opening angle between the leptons (Fig. 2b.) CDF's combined searches in $5.3 \mathrm{fb}^{-1}$ of data [8] and the published search in $4.8 \mathrm{fb}^{-1}$ of data [9] use a Neural network as the final discriminant variable shown in Figure $2 \mathrm{c}$, as does the $5.4 \mathrm{fb}^{-1} \mathrm{D} \varnothing$ analysis [10]. 


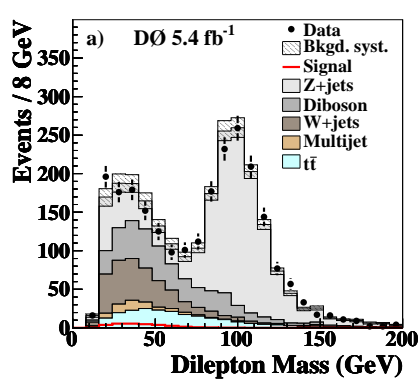

(a)

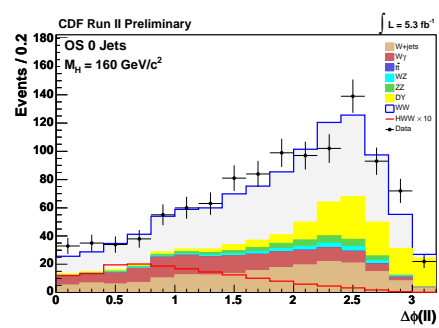

(b)

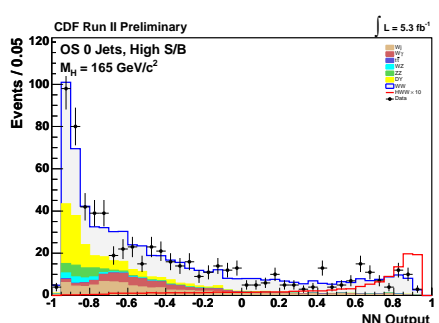

(c)

Figure 2: (a) Di-lepton invariant mass in the $5.4 \mathrm{fb}^{-1} \mathrm{D} \emptyset$ analysis. (b) $\Delta \phi(\ell, \ell)$ in the $5.3 \mathrm{fb}^{-1} \mathrm{CDF}$ analysis. (c) Neural Network output in the $5.3 \mathrm{fb}^{-1} \mathrm{CDF}$ analysis.

\section{Combined Results}

$\mathrm{CDF}$ and $\mathrm{D} \emptyset$ combine their Higgs searches to yield enhanced sensitivity. The most recent combination over entire mass range (100-200 GeV) was in November 2009 [11]. This combination also yields no significant excess and results in a 95\% C.L. observed (expected) upper limit on the cross section of 2.70 (1.78) times the SM cross section at $115 \mathrm{GeV}$, as shown in Figure 3a. CDF and $\mathrm{D} \varnothing$ also combine their most recent published high-mass searches [12] and exclude the SM Higgs boson in the mass range $162-166 \mathrm{GeV}$, shown in Figure $3 \mathrm{~b}$.

\section{Summary}

The CDF and DØ Collaborations perform searches for the Standard Model Higgs Boson in up to $5.4 \mathrm{fb}^{-1}$ of data. The final states include leptons, missing transverse energy, and $b$ jets. No significant excess is observed and upper limits are set on the SM Higgs production cross section in the mass range $100<M_{H}<200 \mathrm{GeV}$ at $95 \%$ C.L., including a combined exclusion of the SM Higgs boson in the mass range $162-166 \mathrm{GeV}$.

\section{References}

[1] LEP Electroweak Working Group, arXiv:0911.2604 [hep-ex] (2009).

[2] The CDF Collaboration, CDF Note 10068 (2010).

[3] The D0 Collaboration, DØ Note 5972-CONF (2009).

[4] The CDF Collaboration, CDF Note 9889 (2009).

[5] The D0 Collaboration, DØ Note 5876-CONF (2009).

[6] The CDF Collaboration, CDF Note 9642 (2009).

[7] V. M. Abazov et al. (D0 Collaboration), Phys. Rev. Lett. 104, 071801 (2010).

[8] T. Aaltonen et al. (CDF Collaboration), CDF Note 10102 (2010).

[9] T. Aaltonen et al. (CDF Collaboration), Phys. Rev. Lett. 104, 061803 (2010).

[10] V. M. Abazov et al. (D0 Collaboration), Phys. Rev. Lett. 104, 061804 (2010). 


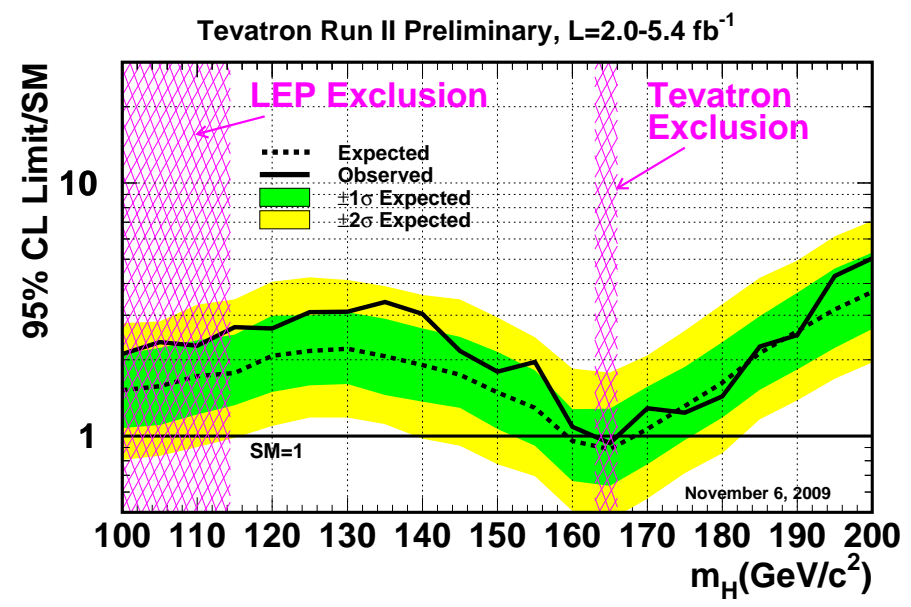

(a)

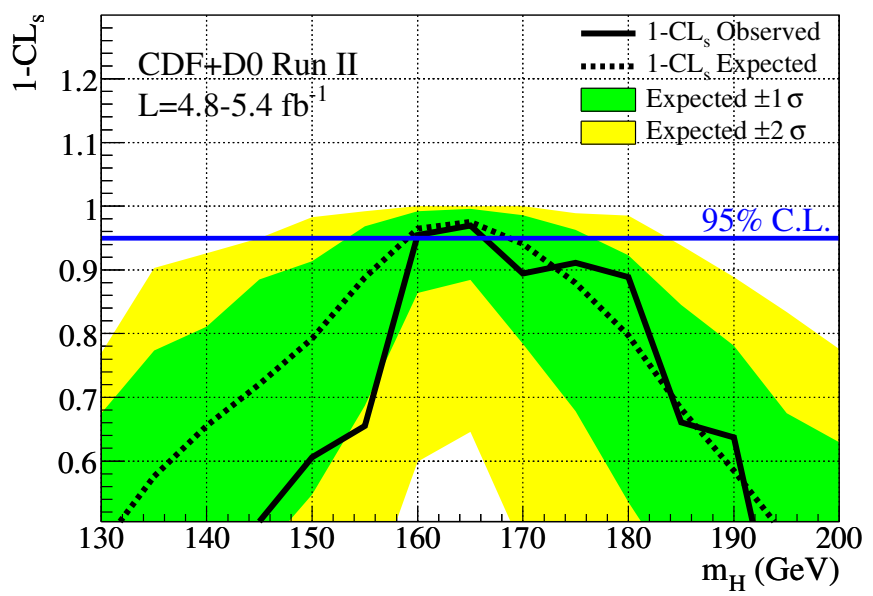

(b)

Figure 3: (a) CDF and DØ combined SM higgs cross section limits as a ratio to the standard model cross section as of November 2009. (b) $1-C L_{s}$ curve [13] for the combined CDF and DØ published $H \rightarrow W W$ searches, showing exclusion at the 95\% C.L. for a Higgs mass between 162 and $166 \mathrm{GeV}$ (b).

[11] Tevatron New Phenomena and Higgs Working Group, FERMILAB-CONF-09-557-E, arXiv:0911.3930v1 [hep-ex] (2009).

[12] T. Aaltonen et al. (CDF and D0 Collaborations), Phys. Rev. Lett. 104, 061802 (2010).

[13] W. Fisher, FERMILAB-TM-2386-E (2006). 